\title{
Projekt DEAL
}

\section{Marcel Knöchelmann* \\ Systemimmanenz und Transformation: Die Bibliothek der Zukunft als lokale Verwalterin?}

https://doi.org/10.1515/bfp-2020-0101

Zusammenfassung: Deutschland bemüht sich, als geschlossener, exzellenter Wissenschaftsstandort durch Rahmenverträge mit Großverlagen einen höheren Anteil an Open-Access-Publikationen zu erreichen. Dies erwirkt lediglich eine Transformation im Sinne einer systemimmanenten, sanften Evolution und verhindert den Gerechtigkeitsgedanken, der Open Access ursprünglich war. Die Zukunft der Bibliothek scheint auf die Rolle der lokalen Verwalterin reduziert zu sein, anstatt eine integrative, tatsächlich systemgestaltende Position einzunehmen. ${ }^{1}$

Schlüsselwörter: Projekt DEAL; Bibliothek der Zukunft; Open Access; Wissenschaftskommunikation

\section{System-Immanence and Transformation: The Library of the Future as Local Administrator?}

\begin{abstract}
Germany as a unified site for science and scholarship aims to enlarge its share of open access publications by means of transformative agreements with large publishers. This, however, is but a transformation in the sense of a soft evolution within the existing system. It prohibits the notion of equity that was once foundational for open access. The future of the library seems to be reduced to the role of the local administrator instead of it being an integrative institution with the ability to change the system.
\end{abstract}

Keywords: Projekt DEAL; library of the future; open access; scholarly communication

1 Ich danke dem Arts \& Humanities Research Council (AHRC), der London Arts and Humanities Partnership und der Studienstiftung des deutschen Volkes für die finanzielle und ideelle Unterstützung meiner Studien sowie der Yale University für die Ermöglichung eines Forschungsaufenthaltes am Department of Sociology, wo die Grundzüge dieses Artikels entstanden.

*Kontaktperson: Marcel Knöchelmann, marcel.knochelmann.15@ucl.ac.uk
Inhalt

1 Einleitung.................... 151

2 Die Zukunft der Bibliothek als integrative

Institution. ................. 152

3 Systemimmanenz und Projekt DEAL . . . . . . . . 153

1.1 Materialistische Problemfelder . . . . . . . . . . . 154

1.2 Ideologische Problemfelder . . . . . . . . . . . . 156

4 Alternativen als Systemtranszendenz. . . . . . . . 158

$5 \quad$ Fazit. . . . . . . . . . . . . . . . . . . . 159

\section{Einleitung}

Der Wissenschaftsstandort Deutschland setzt beim Thema interne Wissenschaftskommunikation auf evolutionäre Transformation. Die Praktiken des Publizierens sollen bürokratisch weiterentwickelt, aber nicht radikal verändert werden. Dieser sanfte Weg hin zu einer für Leserinnen offenen Zukunft verhindert tiefgreifende Änderungen, die notwendig wären, um eine Transformation zu erwirken, die das den Kommunikationspraktiken zugrundeliegende Problem tatsächlich erreichte. Die Idee von einer Zugangstransformation, die durch nationale Rahmenverträge begleitet wird, verstetigt stattdessen die gegenwärtigen Praktiken. Das Projekt DEAL und seine Auswirkungen sind exemplarisch hierfür.

Open Access (OA) gilt als der Modus der Zukunft in weiten Teilen der informationsprokurierenden Institutionen. Dieses OA bringt eine Inversion bibliothekarischer Arbeit mit sich: Wohingegen die frühere Bibliothek physische Informationsmedien aus aller Welt lokal verfügbar machte, muss sich die Bibliothek heute darum bemühen, die lokalen Wissenschaftlerinnen an die digitale Welt anzuknüpfen. Bibliotheken bemühen sich, politische oder überregionale OA-Vorgaben lokal umzusetzen. Projekt DEAL ist zugleich politisch wie auch überregional, sodass lokale Instanzen die operative Hauptlast tragen. Bemerkenswert ist bei vielen solcher Vorgaben und ebenso bei Projekt DEAL, dass sie oft aus dem tradierten System heraus gedacht sind und somit systematisch tradierte Praktiken fortschreiben, lediglich mit rahmenvertraglich 
veränderten Bedingungen. Bezahlschranken und Urheberrechtsbedingungen werden verändert, aber systematisch ändert sich nichts an den Praktiken des Publizierens. Daher sind diese Vorgaben - und Projekt DEAL wieder exemplarisch - als systemimmanent zu bezeichnen. Hier setzt meine Kritik an, denn die Zukunft der Bibliothek wird als eine Zukunft der integrativen Institution gesehen. Die Bibliothek ist keine Akteurin mehr, die lokal ein Profil der Informationsbereitstellung schafft; sie ist integrativ eingebunden in ein Netz aus Institutionen. Die Frage drängt sich auf, ob sie lediglich lokale Verwalterin des überregionalen Netzes ist oder ob sie qua bottom-up-Initiativen das Netz maßgeblich mitbeeinflusst und so ggf. die Vorstellungen und Prinzipientreue des gegenwärtigen Ansatzes an offenes Publizieren überwindet. ${ }^{2}$ Diese zwei Aspekte möchte ich in der folgenden Kritik zusammenführen: Die Systemimmanenz der durch DEAL exemplifizierten OA-Idee wirkt als ein überregionales Netz, in das die Bibliotheken integriert sind. Können sie hierbei mehr sein als lokale Verwalterinnen?

Widerstand gegen DEAL wirkt oft blass, insbesondere die alternativen Initiativen schaffen es materiell oder ideologisch kaum, das tradierte System zu transzendieren oder etwa rhetorisch hohe Wellen zu schlagen. Daher stelle ich die kritische These auf, dass die als Transformation beschworenen DEAL-Verträge abgesehen von der Semantik des transformative agreements nur eine systemimmanente, sanfte Evolution der tradierten Publikationspraktiken darstellen. ${ }^{3}$ Bibliotheken können (oder wollen) kaum alternative Wege gehen und sich dieser Evolution widersetzen. Zwar liegt eine hohe moralische Verantwortung bei den Wissenschaftlerinnen selbst, dieses Wiedersetzen $\mathrm{zu}$ leisten. Und doch gilt, wie Mittler klar benennt: „Es ist nicht die Aufgabe der Bibliotheken, Gewinne weniger Verlage zu sichern, sondern ihre Pflicht, für Forschung und Lehre aber auch für die interessierte Öffentlichkeit die Entwicklung offener, virtueller Wissensräume aktiv zu unterstützen und ihre Funktionsfähigkeit dauerhaft $\mathrm{zu}$ si-

2 Dass es bei der Transformation um mehr gehen muss als um OA, wird im Laufe des Artikels ausgebreitet werden. Probleme, die die Wissenschaftskommunikation umtreiben, gehen zudem weit über $\mathrm{Zu}$ gangsbedingungen hinaus (siehe Abschnitt 5). Es ist schließlich gut möglich, dass die langfristige Zukunft des Publizierens gänzlich anders, ggf. nicht im prinzipienhaften OA, sondern in einer kollaborativen, autorenschaftlich-fragmentierten Infrastruktur liegt, bei der eine Idee der Urheberschaft präsent ist, die derzeit kaum mitgedacht wird. Zur Verdeutlichung, wie so eine Utopie aussehen könnte, bedürfte es eines eigenen Artikels. Stattdessen wird auf die Möglichkeit realer Utopien eingegangen (siehe Abschnitt 4), um Zwischenschritte anzubieten.

3 Kupferschmidt (2019), Max Planck Gesellschaft (2019). chern". ${ }^{4}$ Aus dieser Aufgabe heraus ergibt sich auch eine Aufgabe für Bibliotheken, sich kritisch mit den Möglichkeiten auseinanderzusetzen, die sie als lokale Akteurinnen gegenüber dem übergeordneten Netz haben.

Der Fortgang meiner Argumentation ist wie folgt: $\mathrm{Zu}-$ nächst stelle ich heraus, inwiefern die Bibliothek der $\mathrm{Zu}$ kunft als eine integrative Institution gesehen wird. Dann richte ich den Fokus auf Projekt DEAL und stelle kritisch Problemfelder zur Diskussion, aufgrund derer diese Transformation als eine systemimmanente Evolution zu sehen ist, die zugleich durch Ineffizienz ihr eigenes Wirken verlangsamt. Hernach lege ich kursorisch dar, wie man diese Idee von OA und deren lokale Verwaltung, überwinden könnte. Dabei verfahre ich in keiner der drei Argumentationspunkte enzyklopädisch, sondern kritisch im Sinne einer konflikttheoretischen Auseinandersetzung. Die verwendete Literatur erlaubt bei Bedarf eine darstellende Weiterführung zu den einzelnen Punkten. Dieser kritische Ansatz ist notwendig für den bibliotheks- und informationswissenschaftlichen Diskurs, um auf genau jene Systemimmanenz hinzuweisen, die - wie unten gezeigt wird im Diskurs oft als gegeben aufgenommen wird.

\section{Die Zukunft der Bibliothek als integrative Institution}

Bibliotheken fällt aufgrund ihrer Funktion als Verfügbarmacherinnen die neue Rolle zu, OA zu ermöglichen. Das Verfügbachmachen verschiebt sich somit von der Bestandsentwicklung zu einer Art Integration. Damit liegt OA scheinbar im Trend dessen, was die Bibliothek in Bezug auf Forschung zukünftig insgesamt leisten soll.

So findet etwa Knoche, dass die Hauptaufgabe der wissenschaftlichen Bibliothek morgen wie vor Jahrtausenden die „Verantwortung für die Verfügbarkeit von Veröffentlichungen“ ist - diese Verfügbarkeit jedoch schafft sie nicht mehr allein, sondern nur noch im kollaborativen System. ${ }^{5}$ Die einzelne Bibliothek wird so integraler Teil eines Netzes: Wie die MIT-Bibliotheken es für sich definieren, findet eine Transformation statt von Vergangenheit („knowledge is accessed individually through analog and digital means“) zur Zukunft (,creation and access to knowledge are dynamically networked"). ${ }^{6}$ Erwerbung und Bereitstellung von Medien nehmen funktional immer we-

\footnotetext{
4 Mittler (2018) 24.

5 Knoche (2018) 9.

$6 \operatorname{MIT}(2016) 4$
} 
niger Raum ein, während die Bibliothek sich zu einer vernetzten, digitalen Dienstleisterin entwickelt. ${ }^{7}$ Die Institution kann sich so von der lediglich ,elektronischen Bibliothek zur innovativen Forschungsinfrastruktur" entwickeln und sich ein neues Profil verschaffen. ${ }^{8}$ Sie wird zur Schnittstelle eines größeren, von Daten getriebenen Ganzen: „Die Kunst des Sammelns besteht in der Datenund Dokumentaufbereitung, in der Schaffung von forschungsintegralen Datenzyklen, kurz, in der Datafication und der Entwicklung der Bibliothek als Schnittstelle“.9

Bibliotheken haben also - und bekommen zukünftig noch stärker - die deutliche Prägung als Dienstleistungserbringerinnen. Vornan bei den Empfehlungen der Gemeinschaft britischer Wissenschaftsbibliotheken (SCONUL) ist mithin: „Review local responses to the shift from collections to services in order to position the library effectively in the institution “. ${ }^{10}$ Prägend ist in diesem Satz die Notwendigkeit, lokal Partikularitäten in Bezug auf den Wandel hin zur Dienstleistungserbringerin ausfindig zu machen, da der Wandel letztlich überregional induziert ist. Dieser Effekt ist jedoch keineswegs der Mangel des Fortschritts: Wandel tritt oft supraregional auf und erwirkt so lokal Veränderungen von Praktiken. Die Frage ist aber, wie Bibliotheken mit den local responses umgehen; also: werden lokale Partikularitäten dem Wandel integriert oder werden diese genutzt, um dem Wandel - bottom-up - eine neue Note hinzuzufügen. So würde Fortschritt lokal gestaltet anstatt lediglich verwaltet werden.

Der schwerwiegendste Widerstand, der die lokale Gestaltung aber verhindert, ist die Annahme, dass die überregionale Ideologie gut und richtig und daher mitzutragen ist. Dabei stehen Bibliotheken gar als lokale Akteurinnen mit überregionalen Anbietern in einem neuen Wettbewerb. Dugall drückt das so aus, dass Bibliotheken gar in einem Wettbewerb mit sich entfaltenden Strukturen stehen, bei denen „Open Access ein [...] Paradigmenwechsel [bedeutet], den viele noch gar nicht richtig verstanden haben" ${ }^{11}$ Ob verstanden oder nicht soll - da nicht sachdienlich - hier vorerst nicht relevant sein, ob mitgetragen oder selbst gestaltet, das scheint das viel wichtigere Kriterium.

Dieses Kriterium geht über die Bibliothek hinaus, in Deutschland gar bis zur Hochschulrektorenkonferenz, von wo DEAL wesentliche Richtungsweisung bezieht. So sind also die lokalen Akteure der überregionalen Zielsetzung

7 Neuhausen (2018).

8 Effinger et al. (2019).

9 Stäcker (2019) 309, Herv. i. Orig.

10 Pinfield et al. (2017) 9.

11 Dugall (2013) 95. des Netzes, in das integriert sie agieren sollen, scheinbar ausgeliefert. Sie können Kritik üben oder sich gar desintegrieren. Das hieße also OA und dessen praktische Umsetzung kritisieren oder gar nationale Verträge wie die mit Wiley oder Springer Nature nicht mitzuzeichnen. Was passiert aber, wo über 700 Institutionen zusammengefasst sind und lokal verwalten sollen, was überregional entschieden wird? Faktisch kann jede Einheit Kritik üben und Einwände erheben im Prozess der vertraglichen Netzgestaltung. Aber da OA bereits als Prinzip weitestgehend als das einzig Gute kritiklos akzeptiert ist, kann Kritik nur noch scheinbar Ideologie-immanent stattfinden, also innerhalb der Auslegung, wie die sanfte Evolution zu mehr Offenheit im bestehenden System zu leisten ist. Allen voran nehmen sich - oder lassen sich nehmen - die Bibliotheken so Handlungsspielräume, um tiefgreifendere Probleme der Publikationspraktiken anzugehen oder vor Ort radikalere Lösungen aufzubauen. Nähere Betrachtungen der systemimmanenten, sanften Evolution, verkörpert durch die DEAL-Transformation, sind hierfür aufschlussreich.

\section{Systemimmanenz und Projekt DEAL}

Nationale OA-Verträge sollen einen Rahmen für eine Transformation bieten. Projekt DEAL selbst beschreibt seine Aufgabe damit, dass es „bundesweite transformative ,Publish and Read'-Vereinbarungen mit den größten kommerziellen Verlagen für wissenschaftliche Zeitschriften“ aushandelt. ${ }^{12}$ Diese Rahmensetzung zeigt, dass es sich bei OA lediglich um ein systemimmanentes Verständnis von Offenheit handelt. Das System soll möglichst bleiben, wie es derzeit ist; zugleich sollen aber, wo möglich, die im System geschaffenen Produkte möglichst weit zugänglich gemacht werden, einerseits als ein Zeichen philanthropischer Redistribution, andererseits als ein Mehrwert für die Autorinnen, ihre Institutionen und letztendlich Deutschland als Wissenschafts- und Wirtschaftsstandort. OA wird damit als eine Lösung innerhalb eines Systems gehandelt, bei dem viel tiefgreifendere Probleme bestehen, die systemimmanent nicht so einfach zu lösen sind. OA bietet gar eine Erlaubnis dafür, das System nicht zu verändern: Das moralische Gut wird ausgezeichnet, Wissen zur freien Verfügung zu stellen, es zugänglich zu machen, was ein Aktionismus bewirkt, der nur Kosmetik am bestehenden Sys-

12 https://www.projekt-deal.de/aktuelles/. 
tem ist. Das Problem, das der engstirnige Fokus auf OA bereitet, ist nun dasjenige, dass Handlungsspielräume für eine substanzielle Systemänderung minimiert werden. Das lässt sich daran erkennen, dass zum einen die formalrationalistische Logik der Ergebnisse von Projekt DEAL eine Effizienz vorgaukelt, die es nicht leistet; sowie zum anderen die ideologischen Grundannahmen unverändert bleiben. ${ }^{13}$

\subsection{Materialistische Problemfelder}

Das von Kennziffern getriebene Verlangen nach $\mathrm{OA}$, für das DEAL exemplarisch ist, reiht sich ein in eine Reihe von Praktiken des New Public Management, bei dem Sinn und Zweck von Handlungen von formal-rationalistischer Steuerung (fehl-)geleitet werden. Dies ist bereits in zahlreichen Studien des externen Managements von Wissenschaft herausgearbeitet worden. ${ }^{14}$ Der wiedererkennbare Mechanismus ist hier, dass der nach außen wirkende, formale Rahmen von Handlungen effizient präsentiert wird, wohingegen sich die Handlungen intern hauptsächlich durch Ineffizienz auszeichnen, wie dies von Meyer und Rowan theorisiert wurde. ${ }^{15}$

Ohne zugleich in einen historischen Materialismus verfallen zu wollen, kann man getrost argumentieren, dass das Verlangen nach Offenheit im wissenschaftlichen $\mathrm{Pu}$ blizieren aus der Auffassung materialistischer Ungerechtigkeit heraus erwachsen ist. OA wurde schließlich gesetzt als ein Werkzeug, das wesentlich zur Beendigung der serial crisis fungieren sollte. Diese Krise wurde entfacht und vorangetrieben durch profitorientiertes Wirtschaften, dessen Praktiken hinlänglich bekannt sind. ${ }^{16}$ Kernthese ist und bleibt bis heute: „A concern for many libraries is finding a sustainable way to procure content ${ }^{4}{ }^{17}$ Fraglich ist jedoch, ob es hilfreich ist, mit OA-Großverträgen eine Transformation erreichen zu wollen, die keineswegs ökonomisch nachhaltiger zu sein scheint als die vorangegangenen Abonnementpfade. Man kann schließlich argumentieren, dass das artikelbasierte Preismodell lediglich eine Erweiterung dieser Krise ist. ${ }^{18}$ Wenig nachhaltig ist hier

13 Dieser Dualismus soll nicht bedeuten, dass hier mit zwei Ontologien gearbeitet wird, sondern vielmehr die jeweiligen Problemfelder verdeutlichen.

14 Brink (2018), Collini (2012), Münch (2007, 2008, 2011), Sperlinger et al. (2018).

15 Meyer und Rowan (1977).

16 Baveye (2010), Contreras (2013), Daniels und Thistlethwaite (2016), McGuigan (2004), Muellerleile (2017).

17 Reimer (2018) 1.

18 Khoo (2018) zudem, dass hybride Publikationsmodi teurer sind als andere und dass die als Publish-and-Read gekennzeichneten Verträge hauptsächlich eine Form des Hybriden sind. ${ }^{19}$ Nationale Deals sind, wie Ball andeutet, ,in der Summe noch größer und umsatzstärker als es die alten Big Deals der Subskriptionen waren“. ${ }^{20}$ Woraufhin alsbald der konkrete „Umkehrschluss zum bisherigen Subskriptionsmodell“ erfolgt, bei dem es passieren könne, dass eine Institution keine „Mittel mehr für die Veröffentlichung zur Verfügung hat oder zur Verfügung stellen kann,“ sodass „Wissenschaftler eben nicht publizieren“ könnten, denn die Bezahlung der APCs sei „alternativlos“. ${ }^{21}$ Zwar versuchen DEAL-Verfechterinnen diese Aussagen zu kontern, bestechen aber hauptsächlich mit der rhetorischen Frage dazu, was die Alternative zu Transformationsverhandlungen sein solle: „Weiter Subskriptionen mit altbekannter Preisspirale?“. ${ }^{22}$ Als Antwort kommt hier: „ohne Verträge können Verlage völlig nach Gusto agieren“. ${ }^{23}$ Als gäbe es nur Wissenschaftskommunikation mit Großverlagen oder keine Wissenschaftskommunikation! Eine Antwort könnte stattdessen auch sein, nicht zu versuchen, auf Biegen und Brechen mit dem profitgesteuerten Gebaren von Verlagen klarzukommen, sondern zu ersuchen, die Bedingungen, die dieses Gebaren erst ermöglichen, zu verändern. Bindung von Budgets an DEAL jedoch schreibt die Ermöglichung entgegen aller Veränderung in die Zukunft fort.

Die oft diskutierte und nie richtig gelöste Krise der zu hohen Kosten wird also mit einem auf hohen Kosten basierenden Modell angegangen. Die Rechtfertigung dieser hohen Kosten - und dies knüpft an die Frage der Rechtfertigung der Transformationsverträge insgesamt an - bleibt oft aus. Diffus wird Qualität vorgebracht, dabei ist es ein Truismus, dass Qualität nicht bei den Verlagen hergestellt wird, sondern durch Wissenschaftlerinnen (genau wie auch mangelhafte wissenschaftliche Rigorosität oder Betrug von Wissenschaftlerinnen selbst verursacht wird, was sich Verlage - fälschlicherweise - nicht auf ihre Flyer schreiben). Die Verlage lassen bloß selektieren, was selbst eine systemreproduzierende Praktik ist, da die strenge Hierarchie der Publikationsmarken den Druck zu publizieren und das Produktivitätsmessen anhand von Marken erst befeuert. Verlage rechtfertigen also mit einer Hierarchie, die getarnt ist als Ausweis von wissenschaftlicher Qualität, hohe Kosten, wobei diese Hierarchie auf Qualität erst ein-

19 Knöchelmann (2019a).

20 Ball (2018) 11.

21 Ball (2018) 14

22 Mittermaier et al. (2018) 6.

23 Mittermaier et al. (2018) 6 . 
mal keinen Einfluss hat oder für diese gar nachteilig sein kann..$^{24}$

Daneben gilt es, die Qualität von Technologie infrage zu stellen. Von Verlagen, insbesondere den bei DEAL involvierten, wird Technologie als eine weitere Rechtfertigung hoher Kosten vorgelegt: Datenbanken, Indizes, automatisierte Satz- und Produktionssysteme, die interne Umsetzung externer technischer Standards - all dies muss, so wird proklamiert, produziert und unterhalten werden. Systematisch betrachtet bedeutet dies, dass Bibliotheken einzeln in große Töpfe einzahlen, in denen sodann etwas produziert wird, was den Erhalt der Töpfe rechtfertigt. Und so gelangen wir zu der Perspektive, die das Verlagsargument vom Kopf auf seine Füße stellt. Denn die technische Produktion des immer Neuen, in das stets investiert werden muss, ist ein goldener Käfig, der allen zugutekäme, würden die Geldmittel tatsächlich in kooperative, anstatt in private Töpfe fließen. Die hohen Investitionen, die Elsevier leistet, um Scopus zu betreiben, hätten auch in einer öffentlichen Datenbank resultieren können. Die massiven verlagsseitigen Investitionen in Workflow- und Verlagssoftware hätten auch Open Source sein können - adaptiv und integrativ für alle. ${ }^{25}$ Die Bewegung der offenen Praktiken in der Wissenschaft ist nicht umsonst verwoben mit Open-Source- und Open-Software-Bewegungen. ${ }^{26}$ Was Verlage produzieren - und deswegen ist OA überhaupt erst ein so großes Thema - ist aber immer vorrangig geschlossen.

Die Integration von Lokalem und Netz, die so viele für die Zukunft der Bibliothek halten, ist in Bezug auf große Verlage und DEAL also lediglich die einseitige Nutzbarmachung von Schnittstellen, die teuer erkauft werden, aber nicht abgelehnt werden können, wo Inhalte und Plattformen schlicht nicht mehr getrennt auftreten. Und hier reiht sich ein, dass bei bibliothekarischen Zusammenkünften bereits neuen Monitoringprodukten Gehör verschafft wird, mit denen die lokalen Verwalterinnen ihre bürokratische Integration in den Vertragsfahrplan überwachen können: Es scheint schließlich geschlossener Tools zu bedürfen, die es erlauben, die eigenen institutionellen Publikationsaufkommen und ihre haushälterische Einordnung

24 Brembs (2018), Brembs et al. (2013).

25 Zwar nicht beteiligt an DEAL-Verhandlung, so stellt De Gruyter doch ein aktuelles Beispiel für diesen Mechanismus: Die gegenwärtige Kooperation mit Sheridan PubFactory wird zu einer neuen Plattform führen, die im Jahre 2021 eingeführt wird; zugleich, so hat De Gruyter bekannt gegeben (2020), steht bereits eine neue Kooperation mit 67 Bricks an, die zu einer wiederum neuen Folgeplattform führen wird. Es sind Budgets der Wissenschaft, die diese Investitionen finanzieren. Siehe https://www.degruyter.com/newsitem/406.

26 Tennant et al. (2020). in Bezug auf DEAL zu erschließen und so institutionelle OA-Workflows sichtbar zu machen. ${ }^{27}$ Nicht nur Offenheit wird angeboten, die Analysetools werden von dritten Anbietern gleich mitverkauft! Als finde sich in den über 700 bei DEAL involvierten Einrichtungen keine Instanz, die das praktische Wissen mitbringt, diese nun nicht außerordentlich komplexen Werte des lokalen Publikationsaufkommens automatisiert erfassbar und diese Software open source zugänglich zu machen.

Darüber hinaus treten zuvor kaum geahnte Unstimmigkeiten auf, die einer intensiven Auseinandersetzung bedürfen: Die PAR-Gebühr ist im Falle jeder Publikation zu leisten - Wissenschaftlerinnen können jedoch individuell auswählen, ob sie der OA-Publikation zustimmen oder nicht. Die Transformationsverträge könnten somit in Gänze geschlossene Inhalte produzieren und somit den kleinen Rest ihrer Transformationsnatur in der Praxis verlieren. Wichtig ist daher, sich bewusst zu machen, dass die Verträge nicht OA-and-Read, sondern Publish-and-Read heißen: Für alle Wissenschaftlerinnen gibt es die Opt-OutOption, die bedeutet, dass der Artikel geschlossen publiziert wird, aber für die Institution die gleiche Gebühr anfällt, wie bei einer Hybrid-OA-Publikation. Bereits jetzt macht sich bemerkbar, wie die Kosten von Hybrid am stärksten steigen (so bspw. an der TU Berlin) ${ }^{28}$ und die hehren Ziele von Offenheit der Berliner OA-Vereinigung (Abschnitt 3.2) in ein wertentfremdetes Licht zu rücken drohen: Offenheit muss teuer erkauft werden und ist weit entfernt von einem Ideal der Gerechtigkeit oder transparenten Preisgestaltung. Darüber hinaus ist zu beachten, dass die hohen Kosten der PAR-Fee die noch teureren Hybrid-Produkte der Nature-Journalfamilie nicht miteinschließt. Auch vom Verlag redaktionell beauftragte Beiträge in medizinischen Fachblättern fallen nicht unter die Richtlinie der DEAL-subventionierten Artikel: „Only Research Articles which are not requested and actively editorially managed as part of the editorial program planning, shall be deemed Eligible Articles". ${ }^{29}$ Nicht nur sind diese Feinheiten Distinktionen der Marktmacht auf Seiten Springer (Natures), auch ist dies ein erheblicher Mehraufwand auf Seiten des Bibliothekspersonals, der diese Feinheiten dem wissenschaftlichen Personal in Kleinarbeit zugänglich und auf welchem Wege auch immer verständlich machen muss.

Es wird weiterhin von den Befürworterinnen der nationalen Deals ausgeführt, dass in ,einer Open-Access-

27 Siehe hierzu bspw. Oable: https://oable.org/.

28 TU Berlin (2020).

29 Kieselbach (2020) 16. 
Welt mit CC-BY-Publikationen [...] versteckte[...] Kosten“ entfielen, bspw. die Bezahlung des Abdrucks von Farbabbildungen oder von Überlängen. ${ }^{30}$ Anekdotische Beweisführung der empirischen Realität in Bibliotheken lässt diesen Rückschluss bislang nicht zu. Ganz im Gegenteil muss davon ausgegangen werden, dass versteckte Kosten hinzukommen, die selten der Transformation zugeordnet werden. Nach dem Inkrafttreten der nationalen Verträge müssen diese in die Spezifika lokaler Strukturen bis hinab zur Bereichsbibliothek implementiert werden, wo oft nicht wenige Mitarbeiterinnen in Bibliotheken sich des Themas annehmen. Hier geht es einerseits um die Anpassung der Bezugsstrukturen von Subskription auf PAR-Gebühren inklusive des notwendigen internen Reportings, und andererseits um Vermittlung mit publizierenden Wissenschaftlerinnen bezüglich Optionalität offenen Publizierens, den Schranken des Geltungsraums des Vertrages oder die Aufrechnung weiterer Kosten bzgl. etwa Grafiken. Die Umstellung auf OA auf Seiten der Autorenschaft bedingt letztendlich eine Führungsaufgabe, die mithin von Bibliothekarinnen übernommen werden muss. Genaue Zahlen und Kosten sind bislang unbekannt, doch es ist eine Variable, die durch Aufsummierung schnell zu Substanz kommt. Kursorisches Durchsuchen von Bibliotheksseiten ergibt, dass viele Institutionen bereits mehrere Fachangestellte für OA-Themen bereithalten, oft mit dezidierter Stelle für Publikationsfonds und Transformationsverträge. Es ist offen, ob diese Stellen durch Umwandlung alter, nun obsoleter Stellen oder durch Neubildungen geschaffen wurden. In beiden Fällen sind dies Kosten, die OA und der evolutionären Transformation zuzurechnen sind.

\subsection{Ideologische Problemfelder}

Offenheit und Transparenz werden als ein moralisches Gut der modernen Gesellschaft ausgezeichnet. Sie scheinen bereits semantisch das bessere, korrektere Handeln auszuzeichnen. Ähnlich ist auch OA als ein moralisches Gut verfasst. ${ }^{31}$ Neben dem Diskurs um Open Science - oder Open Humanities ${ }^{32}$ - wurde OA jedoch durch die Vereinnahmung etablierter Akteure in Teilen entfremdet. Die Idee hat sich verselbständigt, OA sei in seiner gegenwärtigen Verbreitung als Publikationsmodus zukunftsweisend, gerecht und daher um jeden Preis geschlossenen Modi vorzuziehen. So wirkt OA in Nordamerika und Europa im

30 Mittermaier et al. (2018) 5.

31 Bacevic und Muellerleile (2017).

32 Knöchelmann (2019b).
Wesentlichen als ein hegemonialer Diskurs. Man muss kein Experte Gramsci'scher Hegemonietheorie sein, um zu erkennen, wie sehr OA ideologische Macht ausübt. Wissenschaftsseitig wie auch verlagsseitig und politisch wird der (falsche) Eindruck erweckt, „mit idealistischen Vorstellungen der Motor einer quasi revolutionären Bewegung für allseitige Offenheit von Wissenschaft im allseitigen gesellschaftlichen Interesse" $\mathrm{zu}$ sein. ${ }^{33}$ Dabei ist das, was DEAL produziert, wie bereits verlautbart, eine Transformation im Sinne einer systemimmanenten, sanften Evolution, die weit davon entfernt ist, revolutionär zu sein.

OA gilt mithin als moralisch notwendig und hat dementsprechend eine politische Tragweite gewonnen, sodass es oft einen wichtigen Platz auf institutionellen Agenden einnimmt. OA ist damit ein Ziel an sich, oft belegt mit prozentualen Werten wie etwa: „Mit dem Jahr 2020 soll der Anteil an wissenschaftlichen Open-AccessPublikationen für Zeitschriftenartikel aus allen wissenschaftlichen Einrichtungen in der Zuständigkeit des Landes Berlin möglichst bei $60 \%$ liegen“. ${ }^{34}$ Dieses Ziel mutet operationalisierbar an, es steht symbolisch für Progress, für eine offene Zukunft. Bei genauerem Hinsehen jedoch entpuppt sich dieser Progress als ein Simulakrum der traditionellen Praktiken, was auf dieses zu erwartende Szenario hinauszulaufen droht: Mit hohem Kostenaufwand wird eine parallele Publikationsstruktur (OA) entwickelt, die den gleichen Konsekrationsmechanismen also dem Übertragen des symbolischen Status des legitimen und qualitätsgesicherten Diskursbeitrages - unterliegt wie die ursprünglichen Praktiken. Diese parallele Struktur hat das Potenzial, die Zugänglichkeitsbedingungen zu Kommunikation aufgrund zusätzlicher ökonomischer Diskriminierung noch zu verschärfen und kommt somit dem hehren Ideal einer Demokratisierung keineswegs näher, wie ursprünglich Befürworterinnen von $\mathrm{OA}$ wie auch die Berliner ${ }^{35}$ oder Budapester ${ }^{36}$ Erklärungen noch vorgaben. ${ }^{37}$ Ganz im Gegenteil scheint OA in seiner derzeitigen, systematischen Implementierung nur wenig dazu beizutragen, epistemische Ungleichheiten abzubauen oder ein dem demokratischen Gedanken gerechteres System hervorzubringen. ${ }^{38}$

Indem Deutschland als Wissenschaftsstandort also scheinbar geschlossen darauf setzt, dass die gegebenen

33 Knoche (2019) 150.

$34 \mathrm{http} / / /$ www.open-access-berlin.de/strategie/index.html.

$35 \mathrm{https}$ //openaccess.mpg.de/Berlin-Declaration.

$36 \mathrm{https} / / /$ www.budapestopenaccessinitiative.org/read.

37 Willinsky (2006).

38 Brembs (2019), Holbrook (2019), Knöchelmann (2020), Sengupta (2020). 
Praktiken der internen Wissenschaftskommunikation richtig sind und lediglich die Bezahlschranke einer Akteursverschiebung bedarf, wird der Illusio des Feldes zugestimmt. Diese besagt, dass eine Hierarchisierung von Publikationen sinnvoll ist und - fälschlich meritokratisch ausgedrückt - Wissenschaftlerinnen diskursive Vormachtstellung und somit Karrierechancen erhalten, so sie nur gute Inhalte vorlegen, deren Qualität man sodann an der Publikationshierarchie ablesen kann. Wie Münch es ausdrückt: „Die Befürworter selektiver bibliometrischer Verfahren nehmen als bare Münze von Qualität, was letztlich sozial konstruiert ist und bei anderen Herrschaftsverhältnissen im akademischen Feld auch anders konstruiert werden könnte“. 39

Dieses ideologische Abonnement ist der wohl schwerwiegendste Schaden, den DEAL verursachen könnte, denn er missachtet die Prozesse der sozialen Schließung wie auch den agonalen Charakter, die durch die Praktiken des Publizierens ausgebildet wurden. Die Exzellenz, die Deutschland für seine Wissenschaft rhetorisch verbucht, kann weiterhin durch Verlagsmarken international konsekriert werden. Der einzige Unterschied ist, dass diese Exzellenz nun noch sichtbarer gemacht werden kann und gegenüber den finanzschwächeren Nationen und Institution philanthropisch umverteilt wird. Exzellenz - diese in der Wissenschaft selbst so unbrauchbare und wertfremde Rhetorik $^{40}$ - kann so noch exzellenter werden; artifizielle Sichtbarkeit und Reputation, noch wichtiger; effiziente Disziplinierung durch standardisierte Qualitätssicherung noch verlässlicher.

OA hätte das Potenzial haben können, radikal mit der Illusio des Feldes zu brechen und eine neue Grundhaltung einzunehmen - es gab dieses Aufflackern des radikalen ideologischen Bruchs. ${ }^{41}$ Auch heute noch gibt es Dissidenten, die Offenheit um eines gerechteren Systems willens fordern (oft aus verschiedenen Positionen heraus) und daher den systemimmanenten OA ablehnen. ${ }^{42}$ Und wenngleich kursorische Nachfragen Kritik an DEAL hörbar machen, ist es schwer, substanzielle Ablehnung aufzufinden, wo in Praxis die Verträge mitgetragen werden.

Die Reifikation von OA als moralischer Notwendigkeit - als eine gesetzesgleiche Gegebenheit - und praktischer Unumgänglichkeit lässt sich an Diskursbeiträgen weiterhin ablesen. Es wird so bereits deklariert, dass „Open Access als Standardmodell des wissenschaftlichen Publizierens“ gelten kann, wo zugleich eine Verlagerung

39 Münch (2007) 192.

40 Collini (2012), Lamont (2009), Moore et al. (2016), Münch (2007).

41 Swartz (2008), Swartz und Lessig (2016).

42 Herb (2018), Moore (2019), Mittler (2018). des „Fokus von Subskriptions- auf Publikationskosten“ stattfindet. ${ }^{43}$ Weiterhin kann man von systemimmanenterfolgreichen Einführungsveranstaltungen lesen, mit denen Naturwissenschaftlerinnen für OA sensibilisiert werden, wie bspw. bei Arndt und Frick. ${ }^{44}$ OA erscheint hier als kritiklos hinzunehmende Maxime, nach der die Zukunft der internen Wissenschaftskommunikation aktivistisch ausgerichtet werden muss - und dementsprechend die Naturwissenschaftlerinnen für diese Zukunft geschult werden müssen. Als Forscherin begegnet man heute an vielen Enden des Systems derart Informationsveranstaltungen, bei denen ein Ablehnen dieser Art OA gleichbedeutend gemacht wird mit Konservatismus; wo also der diskursive Rahmen nur eine Binarität zulässt.

Indikativ für die Stellung, der dieser Modus der Offenheit zugeschrieben wird, ist auch das digitale Abzeichen Open Library Badge ${ }^{45}$ das eher selbstreferentiell als transformativ wirkt. Systemimmanenz wird besonders auch dort deutlich, wo mit vorsichtigen Schritten abgewogen wird, was Vorteile oder Risiken von OA sein könnten, ohne dabei in Betracht zu ziehen, welche Nachteile unter der Kosmetik durch OA bereits vorhanden sind. ${ }^{46}$ Man denke hier nur an die Veröffentlichungspraktiken von Dissertationen in Deutschland: Viele dieser Texte sind Prüfungsmaterial und würden durch eine weiterführende, auf lesendes statt prüfendes Publikum ausgerichtete Bearbeitung erheblich an Mehrwert gewinnen. In genau dieses Wespennest des Pflichtapparats wird aber eher selten diskursiv gestochen. Stattdessen versucht man, noch mehr Dissertationen wie sie als Prüfungstext vorliegen, OA zugänglich zu machen.

Beschreibend erläutert Wohlgemuth ein „Spannungsfeld aus Wollen (Abschluss von Transformationsverträgen mit großen Verlagsgruppen, um eine schnelle OATransformation in der Breite zu erreichen) und Können (begrenzte Budgets der wissenschaftlichen Bibliotheken)“ und impliziert damit, dass Wollen und Können auf diesen Transformationsgedanken fokussiert sind. ${ }^{47}$ Hier könnte man sich an dieses Fokus statt vorstellen, dass das Können zusätzlich dadurch begrenzt wird, dass das Wollen nicht da ist, dass die derzeitige Handhabung von OA nicht als gesetzesgleich und unumgänglich betrachtet wird und Akteure sich vom reifizierten OA befreien: dass also viel eher der Wille zur fundamentalen Veränderung diese Transformation zu transzendieren sucht.

43 Frick und Kaier (2020).

44 Arndt und Frick (2018).

45 Grahl und Wünsche (2020).

46 Graf et al. (2019).

47 Wohlgemuth (2020). 


\section{Alternativen als Systemtranszendenz}

Es gibt also eine andere Lesart der integrativen Bibliothek und die, so schlage ich vor, sieht Institutionen nicht als lokale Verwalterinnen systemimmanenten OA, sondern als Instanzen, die mit ihrer vollwertigen Informationsexpertise aus ihrer Lokalität heraus das übergeordnete Netz verändern. Dies geschieht bereits teilweise, doch verblassen diese Initiativen gegenüber der DEAL-gesetzten Vormachtstellung. Im Sinne einer realen Utopie Wrights könnten bottom-up-Bestrebungen dergestalt verlaufen, sowohl Ideologie zu verändern, als auch alternative Praktiken aufzubauen mit dem Ziel, das gegebene System zu erodieren, wo es nicht mehr zu Revolutionen kommen kann. ${ }^{48}$ So könnten alle Akteure ermächtigt werden, neue Strukturen zu schaffen, die der systemimmanenten, sanften Evolution eine Alternative bieten und so eine viel tiefgreifendere Transformation schaffen.

Bottom up hieße also, dass die Bibliothek als lokale Akteurin lokal Akzente setzt, die sich überregional auf den Wandel auswirken, dass Netzintegration also Netzveränderung involviert. Hierzu braucht die Bibliothek Handlungsvollmacht - oder den Mut gegebene Spielräume aktiv auszunutzen. Dies könnte einerseits negativ erfolgen, indem bspw. die Bibliothek sich nationalen Verträgen verschließt. Dies könnte andererseits auch positiv erfolgen, indem die Bibliothek als die kritische Instanz auftritt und affiliierte Wissenschaftlerinnen nicht lediglich auf vertraglich vereinbarte Möglichkeiten hinweist, sondern Brücken baut hin zu Alternativen. Diese bedeuten, dass es immer auch ohne die großen Verlage gehen kann. Etablierte Publikationsorte sind nur tradiert, sie waren alle einstmals neu, und nie waren die Möglichkeiten zugänglicher als heute, da alle Akteure selbst ein neues Neu platzieren können. Es darf nicht bloß um eine „bedarfsgerechte Hilfe für Wissenschaftlerinnen" gehen; ${ }^{49}$ es muss eine bedarfsverändernde Schulung sein.

Man denke als Beispiel an die Charité, die mit ihren zahlreichen Fachzentren weltweit eine hervorragende Reputation genießt. Diese Reputation hängt nicht von der heutigen Publikation im High-Impact-Journal ab; ganz im Gegenteil bedingt die Reputation, dass Publikationen auch dann gesehen werden, wenn sie auf anderen Plattformen erscheint. Die Charité könnte hier selbst als Konsekrationsinstanz auftreten, indem ihre Wissenschaftlerinnen

48 Wright (2019).

49 Blume (2017), Herv. i. Orig. gemeinschaftliche Publikationsplattformen nutzen und, aufgrund ihrer wissenschaftlichen Reputation, diese Plattform qua Nutzung als relevante Alternative zu den HighImpact-Journals etablieren. Stattdessen ist die Charité bekannt für ihre notorische Metrikbesessenheit; noch immer finden sich Zitationszählungen und die Angabe des Impact Factor auf zahlreichen Bewerbungsseiten der Charité. ${ }^{50}$ Die Ausrichtung der Institution ist weitestgehend Zeugnis des Willens, OA systemimmanent durch Fonds und Repositorien voranzutreiben, die in keiner Weise die kompetitiven und antikollaborativen Praktiken der Erstpublikation ändern. Offenheit wird hier um der Förderung einer individualistischen Exzellenz hin forciert. Und so bleibt die Nutzung des Impact Factor bestehen: „Für die Bewertung von Publikationen soll neben der Relative Citation Ratio (RCR) der NIH und dem Journal Impact Factor der Web of Science Group künftig auch Open Access bei LOM [leistungsorientierter Mittelvergabe] berücksichtigt werden können“. ${ }^{51}$

Ein anderes Experimentierfeld sind Universitätsverlage, die in Deutschland ein eher von Ambiguität als von Tatkraft geprägtes Bild abgeben. ${ }^{22}$ Nach starken Verlagen wie bei britischen oder US-amerikanischen Universitäten - Institutionen, die zwar oft institutionell subventioniert, aber zugleich einen nicht profitorientierten Dienst für die Wissenschaft leisten - sucht man hier vergeblich. Zwar gibt es zahlreiche an Hochschulen angesiedelte, verlagsähnliche Unternehmungen, doch scheinen diese kaum dahin zu streben, als eine Alternative zu den etablierten Verlagen des deutschen Publikationsbetriebes zu Geltung zu kommen. Oft geht es eher um institutionelle Repositorien; angelehnte Verlagsstrukturen verlegen sich auf Abschlussarbeiten oder die gelegentliche hausgemachte Schrift. Gerade starke Zugpferde, Monografien von angesehenen Forscherinnen etwa, die die reputative Kraft haben, Konsekrationseffekte für ein entstehendes Verlagsprogramm auszulösen, findet man kaum. Ein Dilemma diesbezüglich ist aber gerade dieses: Wenn Bibliotheken nicht selbst starke Publikationsinstanzen nach dem Vorbild bspw. von Oxford University Press oder eben des sehr jungen aber erfolgreichen UCL Press werden, dann bleiben sie in einer OA-dominierten Welt darauf verlegt, Gebührenverwalterinnen zu sein. Das Publizieren von Abschlussarbeiten ist mithin nur eine Mehraufgabe, keine Alternative zum Bestehenden.

50 https://promotion.charite.de/en/procedure/regulations_2017/ dissertation/notes_on_journals/, https://mentalhealth.charite.de/e n/research/research_in_numbers/.

51 Flitner und Grimm (2019).

52 Schober (2018). 
Als tatsächlich systemintegrative und kollaborative Praktiken könnte der Aufbau eines OA-Marktplatzes sein, bei dem von Monografien über Journal-Flip bis hin zu individuellen Initiativen OA gemeinschaftlich gefördert werden kann. Mit COPIM wird im internationalen Kontext hierzu bereits gearbeitet. Deutschland scheint sich hier weitestgehend auf Knowledge Unlatched zu verlassen und unterstützt damit den Aufbau von dessen privatwirtschaftlicher Monopolstellung. Mit dem aktuell laufenden Projekt Open Library of Humanities DE gibt es zumindest Anknüpfung an eine kooperative Aufstellung, die in einer Alternative resultieren könnte. Es stellt sich dennoch die Frage, ob das vom Nationalen Open-Access-Kontaktpunkt als Experiment mitgetragene Projekt transcript OPEN Library Politikwissenschaft nicht auch mit einer tatsächlich kooperativen Zusammenarbeit über öffentliche/gemeinschaftliche Strukturen hätte versucht werden können..$^{53}$ Rhetorisch gefragt: Müssen sich Bibliotheken auf geschlossene Intermediäre verlassen, um eine kooperative Struktur $\mathrm{zu}$ testen? Auch hier sehen Bibliotheken mehr wie lokale Verwalterinnen aus, wo Expertise eher an übergeordneten Stellen aufgebaut wird.

Dabei könnten Bibliotheken eine handlungsmächtigere Rolle einnehmen: Wo starke wissenschaftliche Expertise vorhanden ist, können kollaborative und dezentrale Publikationsinitiativen aufgebaut werden, wie das Beispiel von Language Science Press zeigt. Und so wie es FIDs gibt, die zur Förderung des Zugangs existieren, muss es mehr Kollaborationen geben, die eine Förderung alternativer Strukturen wahrnehmen. Wissenschaftliche Institutionen können ihre fachliche Reputation nutzen und so den Neuaufbau einer kooperativen, durch Bibliotheken unterstützten Publikationsplattform konsekrieren. Eben jene Sichtbarkeit, die, so fälschlicherweise angenommen, von Fachzeitschriften hergestellt wird, kann auch durch Beiträge renommierter Wissenschaftlerinnen auf (und für) alternativen Plattformen hergestellt werden. Bibliotheken und dynamische Bibliotheksverbünde können die technische Expertise haben, diesen Aufbau zu begleiten. Stattdessen klammern sich Fachgemeinschaften oft an Publikationsverträge mit Großverlagen, wodurch die Publikationen zumeist geschlossen publiziert oder teuer freigeschaltet werden müssen; bibliothekarische Integration ist hier auf oben benannte Schnittstelle reduziert. Was es braucht, ist der Mut, Systemimmanenz $\mathrm{zu}$ überwinden und mehr sowie radikalere Alternativen zu wagen. Allen voran heißt dies, die Mechanismen, auf denen DEAL basiert, infrage zu stellen und diese Kritik

53 Siehe zum Projekt Jobmann (2018). in Praxis durch alternative Maßnahmen umzusetzen. Sicher, es gibt einige der hier kursorisch angerissenen Alternativen bereits; doch deren Blässe muss mit mehr Mut und Umsetzungswillen in Stärke umgewandelt werden. Die hierfür notwendigen Ressourcen dürfen nicht durch Rahmenverträge beschlagnahmt werden.

Regner und Matthes stellen fest, den „Auftrag das System zu ändern, haben v. a. Forschende, die bereits etabliert sind und sich in gesicherten Positionen befinden “. ${ }^{54}$ Aber das ist nur die halbe Wahrheit, denn die moralische Obliegenheit auf Seiten des wissenschaftlichen Personals braucht praktische Anstöße und Umsetzungsoptionen. Es liegt mithin auch an Bibliotheken mit ihrer „Verantwortung für die Verfügbarkeit von Veröffentlichungen“ ${ }^{55}$ die Wissenschaftlerinnen täglich an diese Obliegenheit zu erinnern und die Möglichkeiten der Umsetzung zu weisen. Aus den Praktiken der Forschung heraus eine Systemänderung zu erwirken ist fast unmöglich, wo institutionelle Trägheit und Routinen Publikationspraktiken oft fest im Griff haben. Bibliotheken können hier den Anstoß leisten, insbesondere dadurch, dass sie die alternativen Strukturen aufbauen und den Weg zu ihnen aufzeigen.

\section{Fazit}

Es hat gar kafkaeske Züge: OA - die Idee von gerechteren Publikationspraktiken, die auf dem hehren Ideal fußt, to "lay the foundation for uniting humanity in a common intellectual conversation and quest for knowledge“56 scheint seiner selbst entfremdet und zugleich ausschließlich besorgt um die sie umgebende Bürokratie. Wie ein ungeheures Ungeziefer findet sich die Forderung nach Offenheit in allen Institutionen, die sodann besorgt sind, messbare Größen der Offenheit zu erreichen. Und zugleich ist die Qualität und Bedeutung, die Offenheit haben sollte oder könnte, völlig aus dem Blickfeld geraten.

Die tiefgreifenden Probleme heutiger Publikationspraktiken werden durch den engstirnigen Fokus auf OA, insbesondere so wie es durch DEAL vorangetrieben wird, nicht gelöst werden. Hierzu zählen allen voran diejenigen Praktiken, die häufig (aber nicht nur) als Publish-or-PerishKultur diskursiv verlautbar werden: hoher Publikationsdruck, der Zwang, strategisch zu publizieren und neben qualitativ hochwertigem reine Masse als Produktivitätsausweis zu leisten; Publikationsorte aufgrund ihrer

54 Regener und Matthes (2019).

55 Knoche (2018) 9.

56 https://www.budapestopenaccessinitiative.org/read. 
Sichtbarkeit und symbolischen Strahlkraft auszuwählen, Erkenntnisgewinn auf möglichst viele Publikationen auszubreiten, Produktivität an formalen Publikationen allein abmessen u.v.m. Durch Fokussierung auf die sanfte, systemimmanente Evolution hin zu OA, scheinen Bibliotheken nicht zur Lösung dieser Probleme beitragen zu können. Zumindest scheinen Aufmerksamkeit und Ressourcen häufig anderweitig gebunden.

Man muss sich also fragen, was nach diesem Transformatiönchen kommen soll: Was machen Bibliotheken nach dem Auslaufen von DEAL-Verträgen? Verlängerungen - also Verstetigung der Evolution - also alles wie bisher nur nun open? Wird das den Budgets und Handlungsspielräumen von Bibliotheken substanziell geholfen haben? Und so ist es zudem ein Mysterium, inwiefern der OA-induzierte Paradigmenwechsel, der nach Dugall aufgrund von Unwissenheit vom Gros der Beteiligten noch gar nicht vollends ermessen wurde, den vielbeschworenen integrativen Charakter der Zukunftsbibliothek auf das Minimum der lokalen Verwaltung reduzieren wird oder Anstoß für einen tiefgreifenden Wandel liefert, bei der die Bibliothek aus ihrer Lokalität heraus auf das Netz und seine Komplexität einzuwirken vermag. Diese Komplexität offenbart schließlich die Schnittstellen an ein Kommunikationssystem, das dermaßen an sozialer, ökonomischer und organisatorischer Größe gewonnen hat, dass es immer wieder neue Dienstleistungen braucht, die Licht ins weitläufige Dunkel bringen. Dass diese Größen lediglich historisch gewachsen und kontingent sind, wird oft nicht behandelt. Zumindest haben Bibliotheken die Expertise und so Möglichkeiten mitzuentscheiden, ob sie vermittels Projekt DEAL Kosmetik am System betreiben oder radikal neue Lichtungen schaffen wollen. Sie müssen nicht die lokalen Verwalterinnen sein, als die sie zurzeit aufzutreten scheinen.

\section{Literaturverzeichnis}

Arndt, Thomas; Frick, Claudia (2018): Getting Scientists Ready for Open Access: The Approaches of Forschungszentrum Jülich. In: Publications, 6 (2), 24. doi: 10.3390/publications6020024.

Bacevic, Jana; Muellerleile, Chris (2017): The moral economy of open access. In: European Journal of Social Theory, 21 (2), 169-88. doi: 10.1177/1368431017717368.

Ball, Rafael (2018): Die Transformation des Publikationssystems zu Open Access und die Konsequenzen für Bibliotheken und Wissenschaft: Ausgewählte Aspekte. In: b.i.t. online, 21 (1), 9-17. Verfügbar unter https://www.b-i-t-online.de/heft/201801-fachbeitrag-ball.pdf.

Barnes, Lucy; Gatti, Rupert (2019): Bibliodiversity in Practice: Developing Community-Owned, Open Infrastructures to Unleash Open Access Publishing. ELPUB 201923 d International Conference on
Electronic Publishing. OpenEdition Press. doi: $10.4000 /$ procee dings.elpub.2019.21.

Baveye, Philippe C. (2010): Sticker Shock and Looming Tsunami: The High Cost of Academic Serials in Perspective. In: Journal of Scholarly Publishing, 41 (2), 191-215. doi: 10.1353/scp.0. 0074.

Blume, Peter (2017): Open-Access-Publikationsdienste für die Wissenschaft. Fortbildungsveranstaltung des DBV-Landesverbands Thüringen. In: o-bib. Das offene Bibliotheksjournal, 4 (4), 249-56. doi: 10.5282/O-BIB/2017H4S249-256.

Brembs, Björn (2018): Prestigious Science Journals Struggle to Reach Even Average Reliability. In: Frontiers in human neuroscience, 12. doi: 10.3389/fnhum.2018.00037.

Brembs, Björn (2019): Scholarship has bigger fish to fry than access. Verfügbar unter http://bjoern.brembs.net/2019/10/scholar ship-has-bigger-fish-to-fry-than-access/.

Brembs, Björn; Button, Katherine S.; Munafò, Marcus (2013): Deep impact: unintended consequences of journal rank. In: Frontiers in human neuroscience, 7. doi: 10.3389/fnhum.2013. 00291.

Brink, Chris (2018): The soul of a university: Why excellence is not enough. Bristol, UK: Bristol University Press.

Collini, Stefan (2012): What are universities for? London, UK: Penguin Books.

Contreras, Jorge (2013): Confronting the Crisis in Scientific Publishing: Latency, Licensing, and Access. In: Santa Clara Law Review, 53 (2), 491. Verfügbar unter https://digitalcommons.la w.scu.edu/lawreview/vol53/iss2/3.

Daniels, Jessie; Thistlethwaite, Polly (2016): Being a scholar in the digital era: Transforming scholarly practice for the public good. Bristol, UK: Policy Press.

Dugall, Berndt (2013): Bibliotheken zwischen strukturellen Veränderungen, Kosten, Benchmarking und Wettbewerb. In: ABI-Technik, 33 (2), 86-95. doi: 10.1515/abitech-2013-0012.

Effinger, Maria; Maylein, Leonhard; Šimek, Jakub (2019): Von der elektronischen Bibliothek zur innovativen Forschungsinfrastruktur. In: BIBLIOTHEK - Forschung und Praxis, 43 (2), 311-23. doi: 10.1515/bfp-2019-2067.

Flitner, Ursula; Grimm, Steffi (2019): Einführung von Open-AccessServices an der Charité - Universitätsmedizin Berlin. Ein Praxisbericht. In: GMS Medizin - Bibliothek - Information, 19. doi: $10.3205 / \mathrm{MBI} 000435$.

Frick, Claudia; Kaier, Christian (2020): Publikationskosten für Zeitschriftenartikel abseits von Open-Access-Publikationsfonds Lost in Transformation? In: o-bib. Das offene Bibliotheksjournal, 7 (2), 1-15. doi: 10.5282/0-BIB/5586.

Graf, Dorothee; Burovikhina, Veronika; Leinweber, Natalie (2019): Zukunftsmodell Monografien im Open Access. In: o-bib. Das offene Bibliotheksjournal, 6 (4), 164-77. doi: 10.5282/O-BIB/ 2019H4S164-177.

Grahl, Tina; Wünsche, Stephan (2020): Open Library Badge. In: o-bib. Das offene Bibliotheksjournal, 7 (1), 1-2. doi: 10.5282/O-BIB/ 5571.

Herb, Ulrich (2018): Open Access and Symbolic Gift Giving. In: Open Divide? doi: 10.5281/zenodo.1206377.

Holbrook, Britt (2019): Philosopher's Corner: Open Science, Open Access, and the Democratization of Knowledge. In: Issues in Science and Technology, $\operatorname{xxv}(3)$. Verfügbar unter https://is sues.org/philosophers-corner-open-science-open-access-andthe-democratization-of-knowledge/. 
Jobmann, Alexandra (2018): Der Nationale Open-Access-Kontaktpunkt OA2020-DE. In: o-bib. Das offene Bibliotheksjournal, 5 (4), 101-12. doi: 10.5282/O-BIB/2018H4S101-112.

Khoo, Shaun Yon-Seng (2018): Article Processing Charge Hyperinflation and Price Insensitivity: An Open Access Sequel to the Serials Crisis. In: LIBER Quarterly, 29 (1), 1. doi: 10.18352/lq.10280.

Kieselbach, Stefan (2020): Projekt DEAL - Springer Nature Publish and Read Agreement. Verfügbar unter https://pure.mpg.de/res t/items/item_3174351_2/component/file_3189424/content.

Knoche, Manfred (2019): Kritik der politischen Ökonomie der Wissenschaftskommunikation als Ideologiekritik: Open Access. 2. Jahrestagung des Netzwerks Kritische Kommunikationswissenschaft. München, 29. November 2019, 140-74. Leipzig University.

Knoche, Michael (2018): Die Idee der Bibliothek und ihre Zukunft. Göttingen: Wallstein Verlag.

Knöchelmann, Marcel (2019a): Open Science in the Humanities or: Open Humanities? In: Publications, 7 (4). doi: 10.3390/publicati ons7040065.

Knöchelmann, Marcel (2019b): Pay to Publish Open Access: On the DEAL-Wiley Agreement. Verfügbar unter https://elephantinthe lab.org/a-groundbreaking-deal/.

Knöchelmann, Marcel (2020): The Democratisation Myth: Open Access and the Solidification of Epistemic Injustices. Verfügbar unter https://osf.io/preprints/socarxiv/hw7at.

Kupferschmidt, Kai (2019): Ground breaking deal makes large number of German studies free to public. In: Science Magazine. doi: $10.1126 /$ science.aaw6836.

Lamont, Michele (2009): How Professor Think: Inside the Curious World of Academic Judgment. Cambridge, MA: Harvard University Press.

Max Planck Gesellschaft (2019): Projekt Deal and Springer Nature reach understanding on world's largest transformative open access agreement. Verfügbar unter https://www.mpg.de/13823 733/projekt-deal-springer-nature-open-access-agreement.

McGuigan, Glenn S. (2004): Publishing Perils in Academe. In: Journal of Business \& Finance Librarianship, 10 (1), 13-26. doi: 10.1300/ J109v10n01_03.

Meyer, John W.; Rowan, Brian (1977): Institutionalized Organizations: Formal Structure as Myth and Ceremony. In: American Journal of Sociology, 83 (2), 340-63. doi: 10.1086/226550.

MIT (2016): Institute-wide Task Force on the Future of Libraries: Preliminary Report. Verfügbar unter https://future-of-libraries. mit.edu/sites/default/files/FutureLibraries-PrelimReportFinal.pdf.

Mittermaier, Bernhard; Holzke, Christoph; Frick, Claudia; Barbers, Irene (2018): Open Access löst nicht alle Probleme - aber mehr, als mancher meint. In: LIBREAS. Library Ideas, (33). doi: $10.18452 / 19282$.

Mittler, Elmar (2018): Open Access: Wissenschaft, Verlage und Bibliotheken in der digitalen Transformation des Publikationswesens. In: BIBLIOTHEK - Forschung und Praxis, 42 (1), 9-27. doi: 10.1515/bfp-2018-0003.

Moore, Samuel (2019): Common Struggles: Policy-based vs. scholarled approaches to open access in the humanities. Verfügbar unter http://dx.doi.org/10.17613/st5m-cx33.

Moore, Samuel; Neylon, Cameron; Eve, Martin Paul; O’Donnell, Daniel Paul; Pattinson, Damian (2016): “Excellence R Us”: university research and the fetishisation of excellence. In: Palgrave Communications, 3. doi: 10.1057/palcomms.2016.105.
Muellerleile, Christopher (2017): Open Access Panacea: Scarcity, abundance, and enclosure in the new economy of academic knowledge production. In: Routledge Handbook of Political Economy of Science.

Münch, Richard (2007): Die akademische Elite: Zur sozialen Konstruktion wissenschaftlicher Exzellenz. Frankfurt a. M.: Suhrkamp.

Münch, Richard (2008): Globale Eliten, lokale Autoritäten: Politik unter dem Regime von Pisa, McKinsey \& Co. Frankfurt a. M.: Suhrkamp.

Münch, Richard (2011): Akademischer Kapitalismus: Zur politischen Ökonomie der Hochschulreform. Berlin: Suhrkamp.

Neuhausen, Hubertus (2018): Die Universitäten digitalisieren sich. In: BIBLIOTHEK - Forschung und Praxis, 42 (3), 411-24. doi: 10.1515/bfp-2018-0060.

Pinfield, S.; Cox, A.; Rutter, S. (2017): Mapping the future of academic libraries: A report for SCONUL. Verfügbar unter http://eprints.w hiterose.ac.uk/125508/.

Regener, Ralf; Matthes, Anja (2019): Open Access in der alltäglichen Diskussion. In: o-bib. Das offene Bibliotheksjournal, 6 (1), 30-41. doi: 10.5282/O-BIB/2019H1S30-41.

Reimer, Torsten (2018): The once and future library: the role of the (national) library in supporting research. In: Insights, 31. doi: $10.1629 /$ uksg.409.

Schober, Claudia (2018): Open-Access-Monografien in deutschsprachigen Universitätsverlagen. In: o-bib. Das offene Bibliotheksjournal, 5 (2), 163-80. doi: 10.5282/0-BIB/2018H2S.

Sengupta, Papia (2020): Open access publication: Academic colonialism or knowledge philanthropy? In: Geoforum. doi: 10.1016/j. geoforum.2020.04.001.

Sperlinger, Tom; McLellan, Josie; Pettigrew, Richard (2018): Who are universities for? Re-making higher education. Bristol, UK: Bristol University Press.

Stäcker, Thomas (2019): Die Sammlung ist tot, es lebe die Sammlung! In: BIBLIOTHEK - Forschung und Praxis, 43 (2), 304-10. doi: 10.1515/bfp-2019-2066.

Swartz, A.; Lessig, L. (2016): The Boy Who Could Change the World: The Writings of Aaron Swartz. New York, NY: New Press.

Swartz, Aaron (2008): Full text of "Guerilla Open Access Manifesto". Verfügbar unter https://archive.org/stream/GuerillaOpenAcces sManifesto/Goamjuly2008_djvu.txt.

Tennant, Jonathan; Agarwal, Ritwik; Baždarić, Ksenija; Brassard, David; Crick, Tom; Dunleavy, Daniel J.; Evans, Thomas Rhys; Gardner, Nicholas; Gonzalez-Marquez, Monica; Graziotin, Daniel; Greshake Tzovaras, Bastian; Gunnarsson, Daniel; Havemann, Johanna; Hosseini, Mohammad; Katz, Daniel S.; Knöchelmann, Marcel; Madan, Christopher R.; Manghi, Paolo; Marocchino, Alberto; Masuzzo, Paola; Murray-Rust, Peter; Narayanaswamy, Sanjay; Nilsonne, Gustav; Pacheco-Mendoza, Josmel; Penders, Bart; Pourret, Olivier; Rera, Michael; Samuel, John; Steiner, Tobias; Stojanovski, Jadranka; Uribe-Tirado, Alejandro; Vos, Rutger; Worthington, Simon; Yarkoni, Tal (2020): A tale of two ,opens': intersections between Free and Open Source Software and Open Scholarship. Verfügbar unter https://osf.io/preprints/socarxiv/2kxq8/.

TU Berlin (2020): Auf dem richtigen Weg: Open-Access-Anteil der TU Berlin bei $50 \%$. Verfügbar unter https://blogs.ub.tu-berlin.de/p ublizieren/2020/05/auf-dem-richtigen-weg-open-access-anteilder-tu-berlin-bei-50/.

Willinsky, John (2006): The access principle: The case for open access to research and scholarship. Cambridge, MA: MIT Press. 
Wohlgemuth, Michael (2020): Artikelbearbeitungsgebühren im Spiegel von Forschung und Praxis. In: BIBLIOTHEK - Forschung und Praxis, 44 (2), 277-82. doi: 10.1515/bfp-2020-2087.

Wright, Erik Olin (2019): How to be an anticapitalist in the twenty-first century. London, UK: Verso.

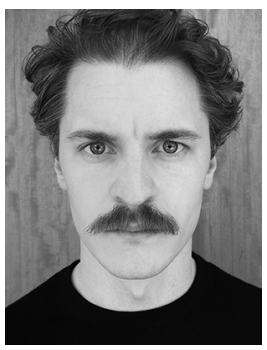

\section{Marcel Knöchelmann}

University College London

Department of Information Studies

Gower Street

London, WC1E 6BT

Great Britian

marcel.knochelmann.15@ucl.ac.uk

Yale University

Department of Sociology

493 College Street, New Haven, CT

USA

marcel.knochelmann@yale.edu 